\title{
The Relevance of Platelet Count and Platelet Lymphocyte Ratio in Sepsis - A Retrospective Study
}

\author{
Alfy Ann George1, Teju Parankimammottil Thomas², Indira Praseeda ${ }^{3}$ \\ ${ }^{1,3}$ Department of Pathology, Travancore Medical College \& Hospital, Kollam, Kerala, India. ${ }^{2}$ Department of \\ Anaesthesia, Travancore Medical College \& Hospital, Kollam, Kerala, India.
}

\section{ABSTRACT}

\section{BACKGROUND}

Beyond being the vital component in maintaining haemostasis, platelets play a vital role in inflammatory conditions and diseases. The aim of this study was to assess the association of platelet counts and platelet lymphocyte ratio (PLR) with severity of sepsis assessed using the Sequential Organ Failure Assessment (SOFA) Score.

\section{METHODS}

Authors did a retrospective study in 208 sepsis patients admitted in the MDICU. The platelet count and platelet lymphocyte ratio were calculated from the blood sample drawn within $24 \mathrm{hrs}$ of admission. The study population was grouped based on platelet count (those with platelet count more than or equal to 1 lakh / cu mm and those with a count of less than 1 lakh / cu mm) and PLR (those with PLR of more than or equal to 200 and those with less than 200). The patients were also grouped based on the SOFA score of less than 10 and those with a score of more than or equal to 10 . The association between these groups were then assessed.

\section{RESULTS}

83 patients (65.4\%) who had SOFA score more than 10 had a platelet count $>1.5$ lakh; whereas 29 patients (35.8\%) who had SOFA score more than 10 had a platelet count $>1.5$ lakh. The number of patients who had both thrombocytopenia and a high SOFA score was only 29 (35.8\%). Similarly, the number of patients who had thrombocytopenia and low SOFA score was 52 (64.2\%). So, there was no correlation between high SOFA score $(>10)$ and thrombocytopenia at time of admission in our study. 45 patients (76.3\%) who had PLR more than 200 had a high SOFA score (> 10), whereas only 67 patients (45 \%) with PLR less than 200 had SOFA score more than 10 . This had a significant correlation with $P$ value of 0.001 . In group with SOFA score less than 10,82 patients (55 \%) had PLR less than 200, whereas in only 14 (23.7 $\%)$ had PLR $>200$. Thus, in our study it was found that sepsis was more severe in patients who had a PLR of more than 200.

\section{CONCLUSIONS}

High platelet lymphocyte ratio calculated at the time of admission is associated with higher SOFA score. A PLR $>200$ showed a significant association with patients with SOFA score $>10$. This is a relatively economical and easily available parameter compared to the other biomarkers.

\section{KEY WORDS}

Platelet to Lymphocyte Ratio, Platelet Count, Sepsis, SOFA Score
Corresponding Author: Dr. Teju Parankimammottil Thomas, Kripa, Parankimammottil, Kizhakketheruvu, Kottarakkara Post, Kollam, Kerala, India.

E-mail: tejupt@gmail.com

DOI: $10.14260 /$ jemds/2021/43

How to Cite This Article:

George AA, Thomas TP, Praseeda I. The relevance of platelet count and platelet lymphocyte ratio in sepsis - a retrospective study. J Evolution Med Dent Sci 2021;10(04):199-202, DOI: $10.14260 /$ jemds/2021/43

Submission 24-04-2020,

Peer Review 23-11-2020,

Acceptance 30-11-2020,

Published 25-01-2021.

Copyright (C) 2021 Alfy Ann George et al. This is an open access article distributed under Creative Commons Attribution License [Attribution 4.0 International (CC BY 4.0)] 


\section{BACKGROUND}

Sepsis is defined as a complex life-threatening syndrome of organ dysfunction due to dysregulated inflammatory host response to overwhelming systemic infection. Sepsis is associated with two phases. First a hyper-inflammatory phase, the systemic inflammatory response syndrome (SIRS), this is followed by or co-exists with a compensatory antiinflammatory phase. This will then lead to multiple organ dysfunction syndrome. Sepsis presents with a clinical continuum of varying severity from SIRS, sepsis and septic shock. (Table 1)

The exact pathophysiology by which an inflammation switches from being locally restricted to systemically spread is not understood properly and is probably multifactorial. There may be direct damage by the pathogen itself and or with the toxic products which contribute to the pathophysiology. The endotoxins are found in the blood of patients and associated with shock and multiple organ dysfunction syndrome. The most common source of sepsis is the respiratory system, followed by genitourinary and abdominal infections. In about half of the patients the source of sepsis is unknown, and the cultures of such patients are negative. The sepsis due to unknown origin is increasing and is associated with higher incidences of multi organ dysfunction and mortality.

The role of platelets in modulating various physiological and pathological responses to inflammatory and infectious conditions is a topic of recent research interest. The immunomodulatory action of platelet is due to the direct cell interaction and indirectly due to the chemical mediators released by platelet. ${ }^{1}$ The main function of platelet is to help in endothelial adhesion and to maintain the vascular integrity of vessels at areas of transmigration. Platelet also helps in recruitment of leukocytes at the site of inflammation. They modulate cytotoxic neutrophil effector function and induce a pro-inflammatory phenotype of neutrophils by modulating their activation, phagocytosis and its oxidative burst. Thereby, platelets contribute to excessive inflammatory host response during sepsis and promote the development and progression of sepsis via their involvement in both inflammation and thrombosis. At the same time, it promotes repair of damaged tissues and inhibit further inflammation. The outcome of the patient depends on the equilibrium between the proinflammatory and anti-inflammatory functions. ${ }^{2}$

Thrombocytopenia is the most frequent coagulation abnormality seen in critically ill patients. The reason for thrombocytopenia is multifactorial. Thrombocytopenia in sepsis can be due either-or combination of decreased production from bone marrow, increased destruction, sequestration of platelets in the micro vessels, immune mediated destruction and haemodilution. Thrombocytopenia has also been found to be an independent risk factor for intensive care unit (ICU) mortality.3,4 Identification of the patients who are at risk of poor prognosis at an early stage is very important for timely and appropriate treatment. There are many scoring systems used in predicting the prognosis in ICU patients. According to the Sepsis-3 guidelines, sepsis is diagnosed by the Sequential Organ Failure Assessment Score, which is organ failure assessment and can predict the risk of mortality. 5

In recent years, there have been several studies which showed the vital role played by platelets and lymphocytes in the inflammatory process. The platelet-to-lymphocyte ratio a novel inflammatory factor has been researched recently as a sepsis severity marker. PLR is found to be a useful indicator of inflammation in a wide spectrum of diseases other than sepsis like acute myocardial infarction, acute kidney injury (AKI), cancer condition like hepatocellular carcinoma and nonsmall cell lung cancer. ${ }^{6}$

\begin{tabular}{|c|c|}
\hline Category & Definition \\
\hline \multicolumn{2}{|l|}{ Previous definitions } \\
\hline $\begin{array}{l}\text { SIRS or (Systemic } \\
\text { Inflammatory } \\
\text { Response Syndrome) }\end{array}$ & $\begin{array}{c}2 \text { of the following: temperature }>38 \text { Celsius or }<36 \text { Celsius } \\
\text { heart rate }>90 \text { beats / min, respiratory rate }>20 / \text { minute or } \\
\text { arterial carbon dioxide }<32 \mathrm{mmHg} \\
\text { White blood cell count }>12 * 109 \text { or }<4 * 109 \text { / L }\end{array}$ \\
\hline Sepsis & SIRS with infection (proven or probable) \\
\hline Severe sepsis & Sepsis with evidence of acute organ dysfunction \\
\hline Septic shock & Sepsis with persistent hypotension after fluid resuscitation \\
\hline \multicolumn{2}{|l|}{ Revised definitions } \\
\hline Sepsis & $\begin{array}{c}\text { Life threatening organ dysfunction caused by a dysregulated } \\
\text { host response to infection }\end{array}$ \\
\hline Septic shock & $\begin{array}{c}\text { Sepsis and vasopressor therapy needed to increase mean } \\
\text { arterial pressure to }>65 \mathrm{~mm} \mathrm{Hg} \text { and lactate }>2 \mathrm{mmol} / \mathrm{L} \\
\text { despite adequate fluid resuscitation }\end{array}$ \\
\hline \multicolumn{2}{|c|}{$\begin{array}{l}\text { Table 1. The Third International Consensus Definitions } \\
\text { for Sepsis and Septic Shock (Sepsis-3) }\end{array}$} \\
\hline
\end{tabular}

The objective of our study was to find the prognostic benefit of the platelet count and PLR derived from the blood sample drawn on the day of admission in sepsis severity assessment.

\section{METHODS}

This study was a retrospective study done in the department of critical care medicine, Travancore Medical College, Kollam. The study period was between June 2016 and May 2017. The study population were adult patients admitted in multidisciplinary critical care ICU with sepsis. The sample size of study population was 208. Convenient sampling was done. Platelet count and the PLR was calculated from the complete blood counts of the blood sample collected at the time of admission. The patients were then grouped based on the platelet count into two groups as those with a count of more than or equal to 1.5 lakhs per cu $\mathrm{mm}$ and one that has count less than 1.5 lakhs per cu mm. Based on the PLR the patients were grouped into one with PLR more than or equal to 200 and the other group with a PLR less than 200. Similarly, the patients were also grouped into two based on neutrophil to lymphocyte ratio (NLR) as those with NLR less than 2.5 and those with NLR more than or equal to 2.5. The SOFA score was used for assessing the disease severity. Patient were grouped into two based on the SOFA score as one group with SOFA score less than 10 and the other group with score of greater than or equal to 10 . The association of platelet count, PLR and NLR with SOFA score were assessed.

Paediatric patients, pregnant women, immunocompromised individuals and patients on steroid therapy for more than 5 days were not included in the study. Required data was collected from the clinical records of patient fitting the criteria.

\section{Statistical Analysis}

Data collected was entered in Microsoft Excel and analysed using SPSS V.16. Descriptive analysis was done by calculating 
frequencies and proportions. Chi square test was used to calculate statistical significance.

\section{RESULTS}

As shown in Table 2, 117 study population (56.3\%) was above 60 years and 59 patients (28.4\%) was between the age group 40 to 60 . Majority of the patients (59.1\%) were males. Female patients were 85 (40.9\%). As shown in Table 3A, among the co-morbidity's hypertension was seen in 137 patients $(65.9 \%)$ followed by diabetes mellitus in 116 (55.8\%) patients. 62 patients $(29.8 \%)$ had coronary artery diseases and 53 patients (25.5 \%) had chronic kidney disease. From table 3B, single organ involvement was seen in 76 patients (42.9\%), two organ involvement was seen in 64 patients (36.2\%). 37 patients $(20.9 \%)$ had more than 2 organs involved. As shown in Table 3C neutropenic sepsis (total WBC count $<4500$ ) was seen in 43 patients $(20.7 \%) .89$ patients $(42.8 \%)$ had total count more than $11,000.76$ patients $(36.5 \%)$ had total WBC count between 4500 and 11,000 . As shown in Table 4 the total WBC count was compared with the severity of sepsis assessed by the number of organs involved was assessed. No significant association was found between the total WBC count and the organ involvement ( $\mathrm{p}$ value $=0.077$ ).

\begin{tabular}{|c|c|c|c|}
\hline & & Frequency $(n=208)$ & Percentage \\
\hline \multirow{3}{*}{ Age Group } & $18-40$ & 32 & 15.4 \\
\hline & $40-60$ & 59 & 28.4 \\
\hline & $>60$ & 117 & 56.3 \\
\hline \multirow{2}{*}{ Gender } & Male & 123 & 59.1 \\
\hline & Female & 85 & 40.9 \\
\hline
\end{tabular}

\begin{tabular}{|cccc|}
\hline & & Frequency & Percentage \\
& Diabetes mellitus & 116 & 55.8 \\
Comorbidities & Hypertension & 137 & 65.9 \\
N = 208 & CKD & 53 & 25.5 \\
& CAD & 62 & 29.8 \\
& CVA & 32 & 15.4 \\
Organ & Single organ involvement & 76 & 42.9 \\
involvement & Two organ involvement & 64 & 36.2 \\
N = 177 & More than two organ involvement & 37 & 20.9 \\
TC Group & $<4500$ & 43 & 20.7 \\
WBC Count & $4500-11000$ & 76 & 36.5 \\
Frequency & $>11000$ & 89 & 42.8 \\
\hline & Table 3 & & \\
\hline
\end{tabular}

\begin{tabular}{|c|c|c|c|c|c|}
\hline \multirow[b]{2}{*}{ TC } & \multicolumn{3}{|c|}{ Organ Involvement } & \multirow[b]{2}{*}{$\begin{array}{c}\text { Chi } \\
\text { Square } \\
\text { Test }\end{array}$} & \multirow[b]{2}{*}{$\begin{array}{c}P \\
\text { value }\end{array}$} \\
\hline & $\begin{array}{c}\text { Single } \\
\text { Organ } \\
\text { Involvement }\end{array}$ & $\begin{array}{l}\text { Two Organ } \\
\text { Involvement }\end{array}$ & $\begin{array}{l}\text { More than } \\
\text { Two Organ } \\
\text { Involvement }\end{array}$ & & \\
\hline$<4500$ & $20(26.3 \%)$ & $9(14.1 \%)$ & $5(13.5 \%)$ & \multirow{3}{*}{8.445} & \multirow{3}{*}{0.077} \\
\hline $\begin{array}{l}4500- \\
11000\end{array}$ & $28(36.8 \%)$ & $24(37.5 \%)$ & $9(24.3 \%)$ & & \\
\hline$>11000$ & $28(36.8 \%)$ & $31(48.4 \%)$ & $23(62.2 \%)$ & & \\
\hline \multicolumn{6}{|c|}{ Table 4. WBC Count and Organ Involvement } \\
\hline
\end{tabular}

\begin{tabular}{|cccc|}
\hline \multirow{2}{*}{ PLT } & \multicolumn{4}{c|}{ SOFA Score } & Total \\
& $<=\mathbf{1 0}$ & $>\mathbf{1 0}$ & $\mathbf{1 2 7}(\mathbf{1 0 0} \%)$ \\
$>=1.5$ lakhs $/$ cu mm & $44(34.6 \%)$ & $83(65.4 \%)$ & $\mathbf{8 1}(\mathbf{1 0 0} \%)$ \\
$<1.5$ lakhs / cu mm & $52(64.2 \%)$ & $29(35.8 \%)$ & $\mathbf{2 0 8 ( 1 0 0 \% )}$ \\
\hline Total & $\mathbf{9 6}(\mathbf{4 6 . 2} \%)$ & $\mathbf{1 1 2}(53.8 \%)$ & Table 5. Platelet Count and SOFA Score \\
\hline \multicolumn{4}{|c}{} \\
\hline
\end{tabular}

\begin{tabular}{|c|c|c|c|c|}
\hline \multirow{2}{*}{ NLR } & \multicolumn{2}{|c|}{ SOFA Score } & \multirow{2}{*}{$\begin{array}{c}\text { Chi Square } \\
\text { Test }\end{array}$} & \multirow{2}{*}{ P-Value } \\
\hline & $<=10$ & $>10$ & & \\
\hline$<2.5$ & $34(70.8 \%)$ & $14(29.2 \%)$ & 15.293 & 0.001 \\
\hline$>=2.5$ & $62(38.8 \%)$ & $98(61.3 \%)$ & & \\
\hline \multicolumn{5}{|l|}{ PLR } \\
\hline$<200$ & $82(55.0 \%)$ & $67(45.0 \%)$ & 16.666 & 0.001 \\
\hline$>=200$ & $14(23.7 \%)$ & $45(76.3 \%)$ & & \\
\hline \multicolumn{5}{|c|}{ Table 6. Comparison of NLR \& PLR with SOFA Score } \\
\hline $\begin{array}{l}\text { Nas cal } \\
\mathrm{nt} \\
\end{array}$ & by chi square & $\mathrm{t}, \mathrm{P}<0.05$ co & dered as statis & \\
\hline
\end{tabular}

In Table 5, 83 patients (65.4\%) who had SOFA score more than 10 was not having thrombocytopenia (platelet count $>1.5$ lakh). Whereas in 29 patients (35.8\%) who had SOFA score more than 10 was not having thrombocytopenia (platelet count > 1.5 lakh). The number of patients who had thrombocytopenia and SOFA score more than 10 was only 29 (35.8\%). The number of patients who had thrombocytopenia and SOFA score less than 10 was 52 (64.2\%). So, there were no correlation between high SOFA score $(>10)$ and thrombocytopenia at time of admission in our study. As shown in Table 6, in patient with NLR ratio > 2.5, 62 (38.3\%) patients had SOFA score less than 10 , but 98 patients (61.3\%) had SOFA score more than 10 . This showed a significant correlation between NLR ratio $>2.5$ and high SOFA score (> $10)$ with a P value less than 0.001 . In patient with NLR ratio < 2.5, 34 (70.8\%) patients had SOFA score less than 10 and 14 patients (29.2\%) had SOFA score more than 10.45 patients (76.3\%) who had PLR more than 200 had a high SOFA score (>10), whereas only 67 patients ( $45 \%$ ) with PLR less than 200 had SOFA score more than 10. This had a significant correlation with $\mathrm{P}$ value of 0.001 . In group with SOFA score less than 10, 82 patients (55\%) had PLR less than 200, whereas in only 14 (23.7\%) had PLR > 200.

\section{DISCUSSION}

Sepsis is a syndrome considered as a dysregulated immune response of host to infection. The immune dysregulation includes various proinflammatory or anti-inflammatory responses that are seen in the different stages of sepsis. ${ }^{7}$ The role of platelets in sepsis pathophysiology has been researched recently.

Thrombocytopenia is one of the most common abnormal blood parameters seen in sepsis, which is often related with platelet consumption. Thrombocytopenia has been associated with higher mortality. The reported incidence of thrombocytopenia has ranged from $22 \%-58 \%$ in various studies. Claushuis et al reported in a large study of 931 patients with sepsis, that patients with a thrombocytopenia at ICU admission had a higher sepsis severity score and increased mortality risk. ${ }^{8}$ Venkata $\mathrm{C}$ in their study found that patients with thrombocytopenia had higher severity of illness scores like Acute Physiology and Chronic Health Evaluation (APACHE) III, more likely to develop major bleeding episodes, higher incidence of acute kidney injury, required prolonged vasopressor support and had a prolonged ICU stay. ${ }^{9}$

A low platelet count may occur early in the course of sepsis. Apart from absolute value of thrombocytopenia, the trend of platelet counts which includes the proportion of the drop in platelet count as well as the non-resolution or persistence of 
thrombocytopenia are found to be strong predictors of mortality in sepsis as well. ${ }^{10}$

Low lymphocytes counts are indicative of a supressed immune and inflammatory response which have been reported with many inflammatory diseases as well as sepsis. ${ }^{11,12}$ Herfferman et al postulated that lymphocytes are vital for the regulation of an adequate inflammatory response. Dysregulation due to excessive apoptosis, cellular exhaustion and downregulation may lead to a deleterious inflammatory state. ${ }^{13}$ Based on these findings, the PLR was suggested as being a novel systematic inflammatory indicator. Platelet lymphocyte ratio is a simple calculated parameter from the absolute platelet count and the absolute lymphocyte count obtained from a venous blood sample. The role of PLR as a prognostic marker was first reported in neoplastic diseases like hepatocellular carcinoma and breast cancer. Similar to our study, Yanfei Shen et al in their study found that patients with high PLR $(>250)$ were associated with increased hospital mortality. ${ }^{14}$ But Zheng et al reported that both high and low PLRs are associated with increased mortality in critically ill patients. 15

It is also seen that the platelets have a higher MPV (Mean Platelet Volume) and PDW (Platelet Distribution Width) in sepsis. The rise in MPV in septicaemia is related to increased production of larger or immature platelets as a reaction of septicaemia related platelet consumption or destruction. The PDW indicates the changes in platelet size and platelet activation. Gao et al. have reported that the platelet count decreased and the PDW amounts increased in patients with sepsis, due to this lead to increased mortality rate. ${ }^{10}$ In the study of Njoroge et al. conducted on 125 cases for the use of haematological parameters for sepsis follow-ups and uncovered that the MPV and PDW escalation was a messenger for mortality. 16

\section{CONCLUSIONS}

High platelet lymphocyte ratio calculated at the time of admission is associated with higher SOFA score. A PLR $>200$ showed a significant association with patients with SOFA score $>10$. This is a relatively economical and easily available parameter compared to the other biomarkers.

\section{Limitations of the Study}

Being a retrospective study, all confounding factors that might have been seen in critically ill patients during the study period could not be controlled. The platelet count can be influenced by other cofounders, such as kinds of cancer, immunological components and drug related thrombocytopenia. Being a retrospective study, it was not able to assess the above factors from the database. Hence, further large prospective trials are required to verify the hypothesis we presented.

Data sharing statement provided by the authors is available with the full text of this article at jemds.com.

Financial or other competing interests: None.

Disclosure forms provided by the authors are available with the full text of this article at jemds.com.

\section{REFERENCES}

[1] Kral JB, Schrottmaier WC, Salzmann M, et al. Platelet interaction with innate immune cells. Transfus Med Hemother 2016;43(2):78-88.

[2] Schrottmaier WC, Kral JB, Badrnya S, et al. Aspirin and P2Y12 inhibitors in platelet-mediated activation of neutrophils and monocytes. Thromb Haemost 2015;114(3):478-89.

[3] Vanderschueren S, De Weerdt A, Malbrain M, et al. Thrombocytopenia and prognosis in intensive care. Crit Care Med 2000;28(6):1871-6.

[4] Hui P, Cook DJ, Lim W, et al. The frequency and clinical significance of thrombocytopenia comlicating critical illness: a systematic review. Chest 2011;139(2):271-8.

[5] Singer M, Deutschman CS, Seymour CW, et al. The third international consensus definitions for sepsis and septic shock (Sepsis-3). JAMA 2016;315(8):801-10.

[6] Kutlucan L, Kutlucan A, Basaran B, et al. The predictive effect of initial complete blood count of intensive care unit patients on mortality, length of hospitalization and nosocomial infections. Eur Rev Med Pharmacol Sci 2016;20(8):1467-73.

[7] Boomer JS, To K, Chang KC, et al. Immunosuppression in patients who die of sepsis and multiple organ failure. JAMA 2011;306(23):2594-605.

[8] Claushuis TAM, van Vught LA, Scicluna BP, et al. Thrombocytopenia is associated with a dysregulated host response in critically ill sepsis patients. Blood 2016;127(24):3062-72.

[9] Venkata C, Kashyap R, Farmer JC, et al. Thrombocytopenia in adult patients with sepsis: incidence, risk factors and its association with clinical outcome. J Intensive Care 2013;1(1):9.

[10] Gao Y, Li Y, Yu X, et al. The impact of various platelet indices as prognostic markers of septic shock. PLoS One 2014;9(8):e103761.

[11] Manzoli TF, Delgado AF, Troster EJ, et al. Lymphocyte count as a sign of immunoparalysis and its correlation with nutritional status in pediatric intensive care patients with sepsis: a pilot study. Clinics 2016;71(11):644-9.

[12] Nunez J, Minana G, Bodi V, et al. Low lymphocyte count and cardiovascular diseases. Curr Med Chem 2011;18(21):3226-33.

[13] Heffernan DS, Monaghan SF, Thakkar RK, et al. Failure to normalize lymphopenia following trauma is associated with increased mortality, independent of the leukocytosis pattern. Crit Care 2012;16(1):R12.

[14] Shen Y, Huang X, Zhang W. Platelet-to lymphocyte ratio as a prognostic predictor of mortality for sepsis: interaction effect with disease severity-a retrospective study. BMJ Open 2019;9(1):e022896.

[15] Zheng CF, Liu WY, Zeng FF, et al. Prognostic value of platelet-to -lymphocyte ratios among critically ill patients with acute kidney injury. Crit Care 2017;21:238.

[16] Njoroge SW, Thompson M, Colon-Franco SM, et al. Clinical utility of hematological parameters to predict sepsis prior to clinical presentation in medical intensive care unit patients. Clinical Chemistry 2014;Suppl 60:83. 